\title{
Desafios da gestão de EAD: necessidades específicas para o ensino científico e tecnológico
}

\author{
Gabriela Trindade Perry* \\ Maria Isabel Timm** \\ Raymundo Carlos Machado Ferreira Filho ${ }^{* *}$ \\ Fernando Schnaid ${ }^{* * *}$ \\ Milton Antonio Zaro
}

\section{RESUMO}

O presente artigo tem como objetivo apontar para a necessidade de uma visão sistêmica de gestão dos processo de educação à distância (EAD), para que todas as atividades que fazem parte deste processo possam ser compreendidas dentro de um conjunto de variáveis dinâmicas e interdependentes. Para isto, faz-se uma revisão da história do desenvolvimento da EAD, sob alguns aspectos tecnológicos e pedagógicos, retomando questionamentos feitos durante esta jornada, seguidos da apresentação de um modelo de sistema de processos e elementos componentes de EAD retirado de literatura. Sugere-se que este seja um possível ponto de partida para iniciar o debate acerca de necessidades de EAD que vão além da escolha tecnológica e do modelo pedagógico. O emprego de uma metodologia estruturada de gestão para os processos de EAD está baseado na suposição de que esta postura permitiria investigações a respeito de aspectos bem específicos e pontuais do sistema, identificando relações entre eles, como, por exemplo, as características das mídias escolhidas em relação às necessidades de treinamento de tutores. Como benefícios adicionais, cita-se: aumento na agilidade dos processos; diminuição de retrabalhos por parte de toda a equipe de planejamento, implantação e gestão de EAD, bem como maior garantia de qualidade.
ABSTRACT
This paper aims at emphasizing the need for a systemic approach for project management in distance education, so that all activities that are integrated into the process could be understood as a set of dynamic and interdependent variables. With this purpose, a review of the history of development of distance education is made, stressing specific technological and pedagogical aspects and putting into perspective discussions made along the research, which is followed by a discussion of distance education processes and elements' model taken from the literature. It is suggested that this could be a starting point for the debate on distance education's needs that are beyond technological and pedagogical models. The use of a structured management methodology for distance education is based on the assumption that this approach would allow specific questions to be dealt with, identifying relations among these questions in aspects such as the characteristics of a given media in relation with tutors' training needs. Additional benefits are the flexibility in the management process, reduction in team work during reevaluation of a course and quality control in distant education.

\footnotetext{
Mestre em Engenharia de Produção (PPGEP-UFRGS) - doutoranda em Informática na Educação (PPGIE-UFRGS) gabrielaperry@hotmail.com - http://www.gabriela.trindade.nom.br - bolsista CAPES.

" Jornalista, doutora em Informática na Educação (PPGIE/UFRGS), Coord. Pesquisa e Desenvolvimento de Tecnologia Educacional e EAD, Centro Nacional de Supercomputação (CESUP) UFRGS.

Mestre em Engenharia (PPGEC - UFRGS), doutorando em Informática na Educação (PPGIEUFRGS).

"*** Pós doutor pela UWA - University of Austrália, Coord. Programa de Pós-Graduação em Engenharia Civil-UFRGS fernando@ufrgs.br

Pós doutor em Engenharia Mecânica pela UFSC, Professor orientador do PPGIE-UFRGS e Coordenador do Laboratório de Biomecânica - UFRGS - zaro@ufrgs.br

V. $4 \mathrm{~N}^{\circ} 1$, Julho, 2006
} 


\section{DA TROCA DE CARTAS ÀS PLATAFORMAS DE GESTÃo}

Tendo suas origens localizadas ao redor da década de 30 do Século XIX, o ensino à distância foi consolidado historicamente, enquanto cultura educacional, em grandes universidades européias, em especial na Inglaterra e na Alemanha, há cerca de três décadas (Peters, 2001). Neste tempo, no Brasil, diversas experiências chegaram a ser concretizadas, em particular através do Instituto Universal Brasileiro e o Instituto Radiotécnico Amador (criados em 1941, com cursos de datilografia e radiotécnica por correspondência) e com o Telecurso de $2^{\circ}$ Grau, lançado pela Fundação Roberto Marinho juntamente com o Sistema Globo de Televisão em 1978 (Kramer, 1999). Ao longo de toda a sua história, este tipo de ensino foi alvo de muita controvérsia, em função de dúvidas a respeito de sua eficiência enquanto estrutura educacional desvinculada da prioridade pedagógica. Nos meios acadêmicos, não faltavam críticas a uma suposta commoditização de cursos à distância anteriores à Internet, porque teriam foco apenas na captação de alunos, apresentavam atores e produtores profissionais para a apresentação de conteúdos e não focalizavam as características e necessidades pedagógicas específicas dos estudantes desse tipo de curso (Noble, 2000).

Ao redor do ano 2000, entretanto, estas dúvidas ficam suplantadas em função da promessa lançada pela disseminação dos computadores e da Internet. No mundo inteiro, a atividade de ensino à distância prenunciou novas possibilidades de acesso à educação, por públicos remotos e dispersos, ao facilitar a troca de mensagens entre professores e alunos, de forma síncrona, através de chats (a única ferramenta disponível para interatividade multiponto, naquele momento), atualmente ampliada pela simultaneidade das modernas ferramentas de compartilhamento e pelas transmissões on-line. Também as formas tradicionais de comunicação assíncronas, características da EAD convencional (troca de correspondência via correio) ganharam novo alento, primeiramente com os e-mails e hoje com um arsenal de possibilidades de contato via web.

Fruto do entusiasmo gerado pela massificação dos novos recursos, fetichizados através da já famosa sigla TIC (Tecnologias de Informação e Comunicação), houve uma verdadeira explosão de interesse no ensino à distância (EAD) via Internet. Em 1999, cria-se, no Brasil, a Uni-Rede, rede de instituições federais do ensino nucleadas pela Universidade de Brasília, em torno do projeto de viabilização de projetos conjuntos de cursos à distância. Pouco tempo depois, o Ministério da Educação do Brasil institucionalizou o EAD, através da criação de uma Secretaria de Ensino à Distância, geradora de importantes fontes de financiamento de projetos, especialmente aqueles destinados à formação de professores, bem como disseminadora de critérios de planejamento, implantação e avaliação da qualidade dos cursos.

Nos últimos cinco anos, praticamente todas as universidades se lançaram ao desenvolvimento de EAD, através de iniciativas individuais de alguns professores, de grupos ou de projetos institucionais devidamente bem apoiados em recursos destinados a infra-estrutura e treinamento de professores. Em geral, as primeiras iniciativas estiveram relacionadas com o desenvolvimento ou experimentos de utilização das chamadas plataformas de gestão de curso, como por exemplo Tel Educ, Web CT, Claroline, entre outras. As plataformas oferecem recursos para que os professores organizem os dados gerais dos cursos oferecidos (nome do curso, informações básicas sobre horários, ementa, bibliografia, etc.); sobre os conteúdos aos quais os alunos devem ter acesso (recursos educacionais como textos, imagens e, atualmente, vídeos, animações, etc.); e sobre o gerenciamento de cada turma de alunos (registro, perfil, emails, notas, agenda, recados, etc.). Também foram disponibilizadas ferramentas de comunicação assíncronas, como ambientes interativos para submissão de mensagens e comentários a outras mensagens, já disponibilizadas por alunos ou professores (fóruns) 
e e-mails específicos dentro do ambiente das plataformas, criação de grupos entre os alunos inscritos, etc.

\section{QUESTÕES PEDAGÓGICO-COGNITIVAS}

Pela sua natureza, as TICs constituem um apoio cognitivo real, mais ergonômico ao raciocínio humano, pela não-lineraridade e mesmo pela redundância de informações (sonoras, visuais, táteis) relacionados ao mesmo conteúdo, o que implicaria em reforço natural à atividade neural de processamento de novas informações. Estas tecnologias também representam um estímulo cultural e carregam uma forte possibilidade de que os professores se debrucem sobre suas próprias práticas, a partir da imersão em um novo universo de possibilidades de representação e apresentação de seu domínio de conhecimento, bem como para a reflexão sobre a multiplicidade de opções das formas como vai desempenhar sua atividade docente.

Possivelmente em razão de ter prosperado em ambiente acadêmico, e de ter na memória as críticas ao sistema anterior, a nova onda de interesse pelo EAD veio acompanhada de fortes - e pertinentes - preocupações quanto às questões pedagógicas relacionadas não somente ao ensino à distância em si, mas às possíveis influências das chamadas TICs mediadas por computador nos processos de ensino-aprendizagem, mesmo no ensino presencial. Nesse clima de entusiasmo e expectativas, as TIC e os novos recursos de interatividade síncrona e assíncrona pareciam prometer mais do que sua própria natureza de instrumentos tecnológicos: seriam, em si, recursos pedagógicos. Em alguns casos, as ferramentas tecnológicas - fossem as plataformas de apoio à gestão dos cursos à distância; os softwares de produção de novas mídias; ou as ferramentas de interatividade - foram consideradas espécies de instrumentos que dariam poder aos planejadores e gestores para propor (ou mesmo impor) novas posturas pedagógicas aos professores que as utilizassem.

Novas soluções deveriam implicar mudanças nas posturas de professores e alunos em relação a seus papéis no processo de ensino e aprendizagem. Se fossem adotadas, as novas ferramentas implicariam quase que de forma inevitável em novas práticas pedagógicas e novos modos de produção e tratamento de informações e conteúdos. A adoção de um ou outro sistema de suporte ao ensino baseado na Web (plataforma) seria, segundo esta abordagem inicial, uma espécie de opção inevitável por um modelo pedagógico diferente do convencional e, portanto, um caminho para uma mudança (idem), mudança esta, basicamente, pautada pela recusa do que seria identificado como uma mera transmissão de conteúdos. A decisão do professor em escolher um ou outro sistema lhe acarretaria a possibilidade de escolher entre reproduzir o modelo de ensino convencional ou adotar iniciativas de mudanças.

Um exemplo desse movimento inicial dos pesquisadores foi a busca de uma suposta plataforma ideal. Várias instituições e grupos de pesquisa lançaram-se ao desenvolvimento de plataformas para disponibilização de seus cursos à distância, muitas das quais, além das facilidades tecnológicas, deveriam ser capazes de mobilizar as intenções pedagógicas dos usuários. Seriam, em tese, capazes de levar o professor - de qualquer área, não importando o nível de exigência do curso - a valorizar a interatividade e a participação ativa de todos os alunos, problematizando a interação, o debate e a busca construtiva de soluções, ao invés da mera apresentação de conteúdos. Seu planejamento, via de regra, tomou como ponto de partida inquestionável de que cursos à distância não podiam simplesmente ser equivalentes a aulas presenciais (expositivas) transmitidas, ou disponibilizadas, independentemente de avaliações sobre a oportunidade - ou a necessidade - de vários cursos tecnológicos (caso das Engenharias) de que boas aulas expositivas não sejam abandonadas, mas, ao contrário, planejadas, aprimoradas e ofertadas em conjunto com materiais didáticos acessíveis, 
precisos e com informações qualificadas, técnicas, necessárias ao aprendizado e ao trabalho profissional (TIMM et al., 2003). Aos poucos, a busca da plataforma ideal foi sendo superada, na prática, pela impossibilidade de que os desenvolvedores da plataforma controlassem o uso que cada professor faz dos recursos ali ofertados, ou mesmo de qualquer outra tecnologia educacional ao seu dispor, seja ela informatizada ou convencional. Mesmo as soluções tecnológicas que impõem interatividade e participação direta do aluno na construção de seu aprendizado podem ter seu uso desvirtuado de princípios pedagógicos estabelecidos no seu planejamento, e podem ser usadas em programas mais dirigidos de aprendizado, dependendo dos objetivos do professor, do nível do curso, dos alunos e mesmo das necessidades do conteúdo (Azevedo, 2005).

Além da apropriação das TICs, há outras questões específicas no EAD mediado por computador. Muitas das questões pedagógicas levantadas pela pesquisa acadêmica que cercou o desenvolvimento do EAD, nos últimos cinco anos, no Brasil, relacionaram-se a questões históricas do ensino convencional, como, por exemplo, a oposição (quase que em termos absolutos) entre transmissão e construção de conhecimentos (TIMM, 2005). Peters (2005) alerta para a importância da análise das questões pedagógicas específicas que se referem ao ensino à distância e não aparecem em outro tipo de situação educacional, como a importância da maturidade dos alunos e da observância de variáveis culturais do local onde ocorre este ensino. Os problemas didático-pedagógicos do EAD, diz este autor, precisam ser identificados e equacionados enquanto problemas de educação à distância, seja ela mediada ou não pelas novas tecnologias informatizadas. O alerta vale para que não se aponha a problemas específicos soluções de natureza generalista - se não reducionista -, ou argumentos que fazem sentido em discussões pedagógicas relacionadas a outros níveis de aprendizado, à educação infantil ou mesmo a diferentes escolas ou culturas de pensamento acadêmico educacional. Moore e Kearsely (1996) apontam elementos relacionados ao sucesso ou fracasso de cursos à distância tão diversos quanto estilos individuais de aprendizagem, fatores sócio-culturais-familiares dos estudantes, grau de maturidade, autonomia e escolaridade formal dos alunos (o autores se referem à existência de alunos-de-risco, tendentes à evasão), relevância dos conteúdos, eficiência do feed-back dos instrutores, entre outros.

A análise realista do papel das plataformas de gestão de EAD, bem como de todas as soluções aportadas pelas TICs ao processo educacional, longe de desanimar, ao contrário, estimula os pesquisadores da área, a buscar novas abordagens para o problema, que continua a desafiar os gestores, que já não estão mais às voltas com o entusiasmo em relação ao EAD informatizado, mas com as necessidades reais de infraestrutura, custos e estratégias de implantação, onde a questão do aprendizado construtivo tem preponderância, mas não é o único item, nem o único responsável por eventuais sucesso ou fracasso de adesão, aprendizado, desempenho ou mesmo motivação dos alunos remotos. Dependendo do nível cognitivo do aluno, de sua maturidade, auto-motivação e independência, é possível que a rapidez e a pertinência do feedback seja mais importante, como um item de satisfação com o curso, do que os esforços em fazê-lo interagir com cada etapa do processo de aprendizado.

Planejar e implantar sistemas de EAD, por exemplo, para estudantes de áreas extremamente exigentes em termos de seus conteúdos, como engenharia, medicina, geologia e outros cursos ligados a ciência e tecnologia ${ }^{1}$, demanda mais do que a instalação e o treinamento no uso de plataformas de gestão, seja esta uma ferramenta

\footnotetext{
${ }^{1}$ A referência é à área de Ciência e Tecnologia por ser a dos autores; não há impedimento que este raciocínio seja transposto à outras áreas.

$4 \longrightarrow$ V. 4 No 1, Julho, 2006
} 
com menos ou mais recursos de interatividade. Também demanda mais do que treinamento de professores para uso de câmeras para transmissão on-line, domínio de salas de chat com múltiplos participantes e estratégias de animação de foros de discussão. Inclui uma análise realista do público-alvo, seus interesses, condições cognitivas e acesso tecnológico, dimensionamento econômico e profissional de recursos humanos para tutoria, com instalações, tempo e recursos destinados a treinamento, produção de material, relativos aos conteúdos e à organização do próprio curso, mídias de vários tipos (item que, em especial, abrange uma gama de necessidades, que vão do design à programação). Inclui necessidades administrativas e gerenciais, de natureza burocrática (registros legais, agendamentos, entre outros) ou tecnológica (rotinas de gestão dos servidores e de produção de back-ups, além de tutoria tecnológica aos alunos e professores). Demanda instrumentos de avaliação que vão da satisfação às exigências de conteúdo complexo, performance de procedimentos, raciocínios diagnósticos e algorítmicos, que dão conta das demandas profissionais de alto nível.

Planejar e implantar sistemas de EAD para as áreas de ciência e tecnologia, em escala minimamente industrial (não restrita a uma experiência isolada e descontínua) demanda que se dimensione, de forma realista, cada uma das expressões que constituem a própria formulação deste problema: planejamento e implantação de sistemas. Para isto, é necessária visão sistêmica, destinada a compreender cada um dos itens que compõem este processo de planejamento e integrá-los de forma dinâmica, buscando descrever (se possível, quantificar, no tempo e na complexidade) da forma mais precisa possível cada uma das suas etapas, suas necessidades específicas dentro de um fluxo de atividades inter-relacionadas. Implica ainda a identificação de indicadores para cada etapa, desde a caracterização (cognitiva e de acesso tecnológico) do público-alvo até a avaliação final dos alunos e do curso, passando pela produção do material didático, o treinamento dos professores, de tutores tecnológicos e tutores pedagógicos, dimensionamento de infra-estrutura física, humana e tecnológica, implantação e acompanhamento de rotinas docentes e administrativas, entre outros inúmeros itens de maior ou menor peso no processo, mas igualmente necessários para permitir ao gestor a identificação de falhas, gargalos ou soluções ineficientes, naquela parte específica do sistema onde ocorrem. Tais procedimentos devem levar à superação das respostas simples para perguntas complexas, relacionadas a possíveis causas de evasão. $\mathrm{Na}$ verdade, todos os itens citados - e inúmeros outros - são necessários e fundamentais para que o ensino à distância mediado pela Internet realmente seja uma realidade viável, compatível com a necessidade de excelência do ensino científico e tecnológico, confiável, auto-sustentável, desejável e necessário, que cumpra a promessa tecnológica de aprendizagem democratizada, facilitada, ativa-interativa-colaborativa. A percepção da complexidade desse conjunto dinâmico deve contribuir para tornar mais realista e menos ingênua a suposição de que novo EAD informatizado possa ser um impulsionador de soluções fáceis, implantado sem esforços ou investimentos, com resultados e lucros rápidos.

Hoje, passado o primeiro momento de adesão incondicional à EAD informatizada, a profissionalização da gestão deve levar à busca de soluções que não se atenham à crítica à mercantilização da oferta de cursos, ou à busca de modelos pedagógicos ou soluções tecnológicas ideais, aplicáveis a qualquer área, sem risco[1]. Estas soluções passam pela identificação e quantificação precisa de necessidades, pela compreensão de variáveis que compõem o conjunto do sistema, pela integração flexível dessas variáveis, pela busca de soluções criativas e viáveis economicamente. É preciso construir soluções que dêem conta das questões que inter-relacionam (positiva ou negativamente, dependendo do caso) as necessidades do ensino de conteúdos 
complexos com a economia de escala envolvida na gestão de EAD. Soluções que passam pela identificação e quantificação precisa das variáveis que compõem este sistema complexo, do qual fazem parte, por exemplo, os custos precisos de cada etapa produção (incluídas estratégias de marketing para captação de estudantes remotos), e de cada aluno, para que o curso seja auto-sustentável; os softwares e as mídias instrucionais capazes de vencer a complexidade dos conteúdos científicos e tecnológicos; a discussão sobre a eficiência da implantação e gestão de repositórios de objetos educacionais integrados às plataformas de cursos; a necessidade de estruturação de redes de ensino, através da cooperação entre universidades, ou com o setor produtivo, para viabilizar tutorias eficientes e confiáveis; a compreensão da real necessidade de treinamento e gestão de tutores dos cursos remotos, em áreas que envolvem riscos, custos e confiabilidade; a alocação de tempo para preparação de material nas planilhas de horários dos professores, seja ele mesmo o produtor ou o interlocutor da equipe de mídias. Enfim, soluções que passam pela integração flexível destas e de outras variáveis a serem identificadas, através de modelos de gestão que apontem soluções criativas e viáveis economicamente. Neste sentido, a visão da Engenharia de Produção pode ter uma importante contribuição.

\section{GESTÃO DE PROCESSOS}

Conforme exposto, há grandes dúvidas e grandes expectativas em relação ao potencial das TICs como apoio à EAD. Esta postura tem como consequiência a proliferação de programas, alguns bem-sucedidos, outros não, seja em termos educacionais ou mesmo de sua auto-sustentabilidade. Sugere-se que, até agora, a maior parte deles ${ }^{2}$ tenha contemplado questões muito específicas das diversas disciplinas que integram o contexto multidisciplinar da gestão de EAD, ora de caráter pedagógico, ora de caráter tecnológico. Acredita-se que este posicionamento - de pesquisa e de gestão frente aos inúmeros desafios colocados, apenas resolvem problemas pontuais, sem apontar para a necessidade do aporte de outras áreas do conhecimento - como a gestão de processos -, nas investigações, que darão suporte a futuros projetos que possam compreender o sistema como um todo, para melhor compreender a inter-relação das partes. Assim, a comunidade envolvida com EAD está à frente do desafio de compreender seus processos. Nesta visão, os processos administrativos, por serem reguladores, são onipresentes, tentando minimizar o impacto da burocracia e da regulação vertical.

Moore e Kearsely (1996) de forma muito didática colocam o exemplo do surgimento e evolução das companhias aéreas, cujo funcionamento, em um primeiro momento, restringia-se ao piloto, que encontrava os passageiros na pista, cobrava a passagem e os embarcava, com o auxílio de um único assistente. Hoje, ninguém mais tem dúvidas sobre a necessidade da especialização do trabalho das companhias aéreas e do cumprimento de etapas especificadas e bem divididas. O resultado desta evolução é a provisão de um serviço de alta qualidade com um custo por passageiro dimensionado claramente em função das necessidades de sustentabilidade da empresa. Estendendo a analogia aos cursos à distância, os autores propõem que este apenas se torna viável economicamente quando se beneficia de uma economia de escala: quanto maior o número de usuários, menor o custo por usuário ${ }^{3}$. Acrescenta-se a este argumento o fato que uma visão sistêmica da EAD permite coordenar os esforços para responder também

\footnotetext{
${ }^{2}$ A continuidade da presente pesquisa aponta para estudos de casos específicos de programas de EAD, implantados no Brasil e no exterior, junto a instituições públicas e privadas.

${ }^{3}$ Possivelmente, no Brasil, o dimensionamento claro dos custos de cada elemento que compõe o sistema e mesmo de cada aluno remoto não tenha sido ainda exigido, em função de que muitos programas de EAD são montados a partir de projetos, financiados por órgãos de fomento ligados aos governos federal ou estadual.

6 - V. 4 No 1, Julho, 2006
} 
às perguntas diretamente relacionadas aos aspectos educacionais, estabelecendo, inclusive, relações entre os resultados encontrados nos vários processos de aprendizagem e das relações inter-pessoais que fazem parte do processo, desde a capacitação de professores, dos tutores, dos administradores e dos produtores de mídias.

Assim, apresenta-se o modelo proposto por Moore e Kearsely (1996) na figura 1. Nele é clara a sistematização em etapas, cada uma com seus elementos constituintes, objetivos e particularidades, cada uma das quais, por sua vez, implicando as respectivas atividades, necessidades, métodos de realização, avaliação e identificação de indicadores. Embora seja um modelo extremamente simplificado ${ }^{4}$, de uma publicação de 1996 (ao qual se acrescentaram observações feitas pelos autores, com base na prática de pesquisa e implantação e experiências de EAD), acredita-se que pode ser um ponto de partida para pensar a gerência dos processos de EAD de uma forma global e sistêmica. Sugere-se, por exemplo, que o último item apresentado pelos autores (Ambiente de aprendizagem) poderia estar melhor posicionado no início do processo, como um dos condicionantes das necessidades dos estudantes, que vai, por sua vez, gerar necessidades no projeto do curso, na escolha e na entrega das mídias. O outro quadro aposto ao modelo indica um exemplo de atividades que fariam parte de uma única função na equipe: os administradores ou gestores.
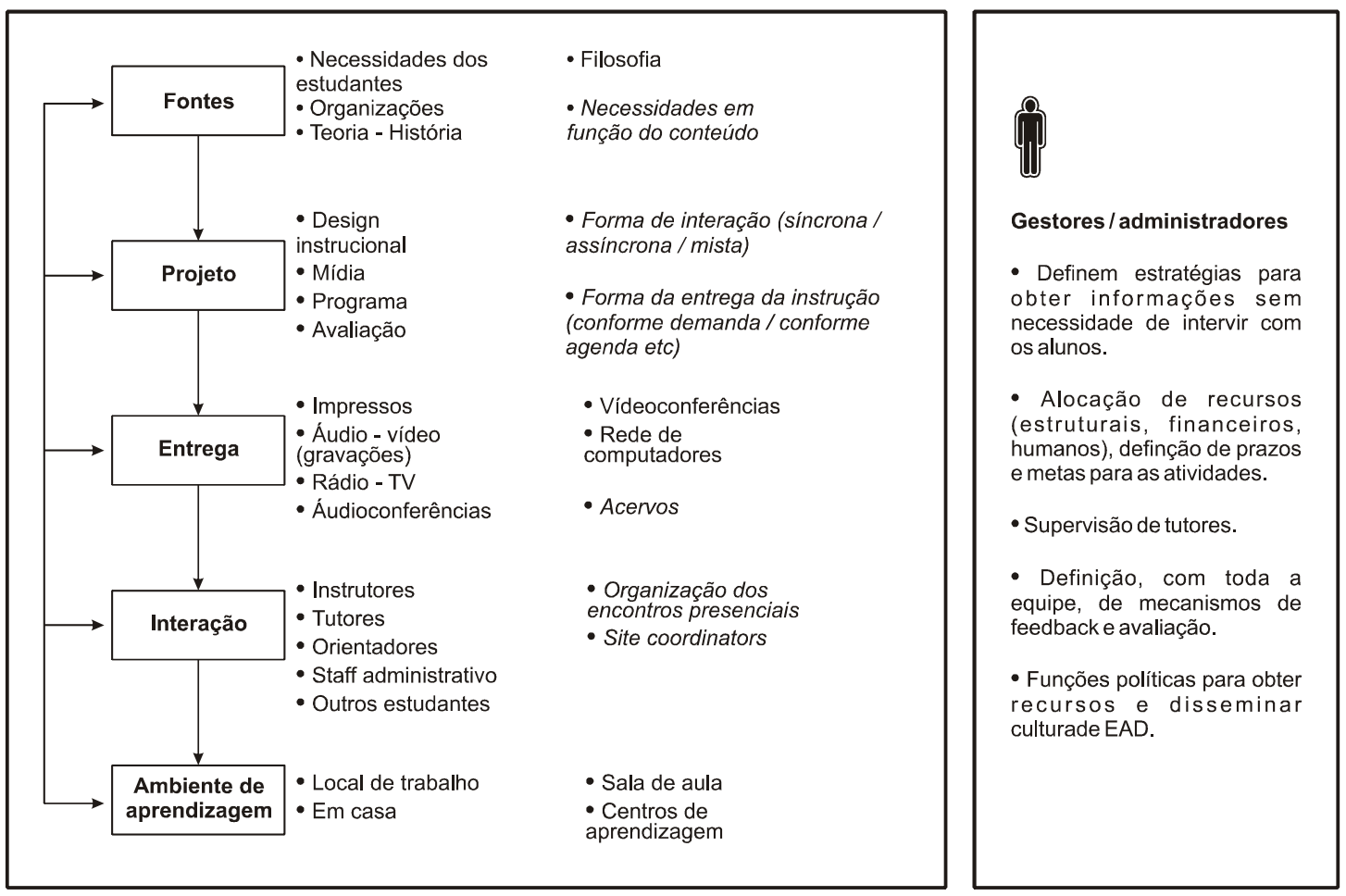

Figura 1 - Modelo de processo para EAD. Retirado de Moore \& Kearsley (1996). Os textos em itálico a o quadro da figura dos gestores/administradores representam inserções feitas pelos autores deste artigo.

O fato de estabelecer uma sistematização para os trabalhos é um dos pontos fortes deste modelo, pois a partir daí é possível pensar nos profissionais que comporiam as equipes e nas atividades delegadas a cada um (o que foi feito a título de exemplo,

\footnotetext{
${ }^{4}$ Questões de marketing, como posicionamento da marca, por exemplo, não são mencionados. Esta é uma das variáveis que pode definir os rumos de todo o curso. V. $4 \mathrm{~N}^{\mathrm{o}} 1$, Julho, 2006
} 
para o gestor). Outro benefício de partir de um modelo como este é sua horizontalidade, o que mostra o compromisso em agilizar as tomadas de decisão que vão ocorrer ao longo de todo o processo e permitir o emprego do conhecimento tácito/empírico dos profissionais envolvidos em cada atividade, diminuindo a carga burocrática e formal. Como exemplo da pertinência e contemporaneidade do assunto, cita-se Smith (2006), diretor do departamento de desenvolvimento profissional em engenharia da Universidade de Wisconsin-Madison, que afirma que a organização em equipes de produção e o estabelecimento de benchmarks e de revisões de qualidade são a regra; van Aalst e van der Mast (2003), que expressam sua preocupação em relação à qualidade da comunicação entre membros da equipe produtora de materiais educacionais, e van der Mast (1995) e Retalis (1997), sobre aspectos da gerência de produção de material.

Certamente a necessidade de investigar questões específicas de cada atividade permanecem. São necessários parâmetros para escolher o tipo de mídia mais adequado; para determinar o momento e a forma de conduzir uma avaliação; para estruturar o currículo; para determinar o tamanho de cada unidade de instrução, etc. Enfim, muitas questões precisam ser respondidas.

Contudo, acredita-se que encarar as práticas de EAD com um olhar sistêmico cria um ambiente propício para as mais diversas investigações, por situar todas as problemáticas dentro de um contexto maior. Um benefício adicional - não menos importante - que pode ser citado é o fato que organizar as práticas e ferramentas de gerência traz como resultados: (a) um aumento na agilidade dos processos; (b) a diminuição de retrabalhos; (c) maior garantia de qualidade; (d) possibilidade de reproduzir e estender práticas e resultados e (e) distribuir, reaproveitar e acumular conhecimento dentro da instituição/comunidade de pesquisa.

\section{CONCLUSÕES}

As questões que levam à preocupação com o tema gestão de processos em EAD são facilmente justificadas. Neste artigo, sua defesa foi estruturada sobre dois eixos: $\left(1^{\circ}\right)$ a necessidade de agregação de valor dos conhecimentos de gestão às pesquisas e aos programas já implantados de EAD mediados por tecnologias de informação e comunicação, uma vez que muitas instituições se dedicaram ao tema apostando na facilidade trazida por estas novas tecnologias, sem dimensionar de forma realista todas as necessidades agregadas ao ensino à distância em si e à nova base tecnológica; $\left(2^{\circ}\right)$ a fruição de benefícios práticos da gestão de processos de educação à distância, dimensionados de acordo com as possibilidades e as necessidades de cada instituição, com a otimimização dos recursos investidos.

No entanto, se tem consciência que este implantar tais processos, de forma sistemática, com indicadores precisos e coleta de informações eficiente é um desafio gigantesco, que tem incontáveis desdobramemtos e níveis de profundidade. Some-se um sem número de dificuldades operacionais (característicos de grandes projetos de software, dos processos educacionais e da própria cultura brasileira, entre outros); a mistura de questões ligadas à investigação científica e ao domínio da técnica; as dificuldades de uma investigação que envolve diferentes áreas (multi e interdisciplinaridade); e a falta de um corpo de conhecimento organizado sobre o tema.

Além do fato de que EAD mediado por computadores é uma área relativamente recente, em termos de toda a história da EAD convencional, acredita-se que estes sejam motivos para o pequeno volume de pesquisas com este enfoque, pois apontariam para a necessidade de uma grande - e desanimadora - quantidade de investimentos, infraestrutura de porte e uma equipe multidisciplinar capacitada para lidar com cada um dos processos. O outro motivo pode estar relacionado com a consolidação desta área junto à comunidade acadêmica, que priorizou - com toda o mérito e razão - a garantia de que o 
novo modelo de EAD não deveria repetir experiências anteriores, criticadas pela excessiva comercialização, sem preocupações pedagógicas e metodológicas. Sugere-se que, hoje, esta mesma comunidade está madura e confiante no caminho percorrido até então nesta área, apontando-se a necessidade de que os esforços se dirijam também aos processos de planejamento e gestão, os quais também terão impacto no aprendizado, na motivação de alunos e professores e nas taxas de evasão dos cursos. Nesse sentido, apontou-se o modelo proposto por Moore e Kearsely (1996), para, a partir dele, apresentar o tema para discussão e futuro refinamento.

\section{Referências Bibliográficas}

AZEVEDO, A.M.P. Tese de doutorado, defendida no Programa de Pós-Graduação em Informática na Educação, UFRGS, sob orientação do professor Milton Antonio Zaro, defendida em 2005

KRAMER, A. et al. Educação à distância: da teoria à prática. Porto Alegre:

Alternativa, 1999.

NOBLE, D. (reproduzindo informação do jornal Le Monde Diplomatique, sem referência de data), In: Jornal AD Verso, da Associação de Docentes da Universidade Federal do Rio Grande do Sul (ADUFRGS), edição de maio 2000.

PETERS, O. Didática do ensino à distância. São Leopoldo: Unisinos, 2001.

PETERS, O. Learning and Teaching on Distance Education. Disponível em $<$ http://fernuni-hagen.de/ZIVV.v2-ch40a.htm> Acesso em 20 dez 2005.

TIMM, M. I. et al. Tecnologia educacional: mídias e suas linguagens. RENOTE: Revista Novas Tecnologias na Educação, Porto Alegre, v. 1, n. 1, p. 1- 19, fev. 2003.

VAN AALST, J. W.; VAN DER MATS, C. Performer: an instrument for multidisciplinary courseware teams to share knowledge and experience. Computers \& Education, 41, p. 39-48, 2003.

TIMM, M. I. Elaboração de projetos como estratégia pedagógica para o ensino de Engenharia (curso à distância de projeto no modelo e-learning-by-doing), tese de doutorado sob orientação do prof. Fernando Schnaid, Programa de Pós-Graduação em Informática na Educação, Universidade Federal do Rio Grande do Sul, Porto Alegre, Brasil, defendida em abril/2005.

SMITH, T. UW Engineers Efficient Quality Control. Distance Education Report. Disponível em <http://www.magnapubs.com/issues/magnapubs_der/cgibin/udt/im.display.printable?client_id=magnapubs_der\&story_id=598730 >. Acesso em 23 mai 2006.

MOORE, M. G.; KEARSELY, G. Distance Education: Systems View. Wadsworth Publishing Company, 1996.

VAN DER MAST, C. Professional Development of Multimedia Courseware. MachineMediated Learning, 5 (3 \& 4), 269-292, 1995. 
RETALIS, S. A courseware development meta-methodology for Open and Distance Learning. In: CAISE'97 - 4th Doctoral Consortium on Advanced Information Systems Engineering, Barcelona. Disponível em <http://www.unikoblenz.de/fb4/publikationen/gelbereihe/RR-14-97/>. Acesso em 25 mai 2006 